\title{
Propofol Inhibits Thyroid Cancer Cell Proliferation, Migration, and Invasion by Suppressing SHH and PI3K/AKT Signaling Pathways via the miR-141-3p/BRD4 Axis
}

\author{
Heming Zhang, ${ }^{1}$ Mingtao Tan, ${ }^{2}$ Jing Zhang, ${ }^{3}$ Xiao Han, ${ }^{4}$ and Yue Ma ${ }^{5}$ \\ ${ }^{1}$ Department of Anesthesia Surgery, Jinan Seventh People's Hospital, Jinan 250101, Shandong Province, China \\ ${ }^{2}$ Department of Anesthesiology, Zibo Central Hospital, Zibo 255036, Shandong Province, China \\ ${ }^{3}$ Department of Anesthesia Surgery, Zibo Central Hospital, Zibo 255020, Shandong Province, China \\ ${ }^{4}$ Department of Nuclear Medicine Radiotherapy, Zibo Central Hospital, Zibo 255036, Shandong Province, China \\ ${ }^{5}$ Department of Anesthesiology, Affiliated Hospital of Hebei University, Baoding 071000, Hebei Province, China
}

Correspondence should be addressed to Yue Ma; mayuevivian2021@163.com

Received 28 October 2021; Revised 10 November 2021; Accepted 23 November 2021; Published 16 December 2021

Academic Editor: Kalidoss Rajakani

Copyright ( 92021 Heming Zhang et al. This is an open access article distributed under the Creative Commons Attribution License, which permits unrestricted use, distribution, and reproduction in any medium, provided the original work is properly cited.

\begin{abstract}
Objective. This study explores the effect and mechanism of propofol for thyroid tumor. Methods. Culture human normal thyroid cells Nthy-ori 3-1 and thyroid cancer cell line TPC-1. TPC-1 cells were divided into the propofol group (treated with propofol), miR-141-3p group (transfected with the miR-141-3p mimic), negative control group (transfected with miR-NC), miR-141$3 p+p c D N A-B R D 4$ group (transfected with the miR-141-3p mimic and pcDNA-BRD4), miR-141-3p + pcDNA group (transfected with the miR-141-3p mimic and pcDNA), siBRD4 group (transfected with siBRD4), and si-control group (transfected with sicontrol). The detection of miR-141-3p and BRD4 expression in cells was done by RT-qPCR, and the dual-luciferase reporter gene method and western blotting were used to verify the targeting relationship between miR-141-3p and BRD4. MTT method was used to test cell proliferation, transwell method was used to test cell migration and invasion, and western blotting was used to test $\mathrm{SHH}$, GLI1, p-PI3K, and p-AKT protein expression. Results. Compared with Nthy-ori 3-1 cells, the expression of miR-141-3p in TPC-1 cells was markedly decreased. Propofol treatment and excessive expression of miR-141-3p could influence the phenotype of TPC1 cells. BRD4 is one of the target genes of miR-141-3p, and its expression is negatively regulated by miR-141-3p. Overexpression of BRD4 can partially reverse the restraining effect of miR-141-3p on the TPC-1 cell phenotype. Both miR-141-3p and BRD4 can regulate the activity of SHH and PI3K/AKT signaling pathways. Conclusion. Propofol can inhibit the activity of SHH and PI3K/ AKT pathways by targeting downregulating BRD4 through miR-141-3p, thereby inhibiting the phenotype of TPC-1 cells.
\end{abstract}

\section{Instruction}

Thyroid cancer, including papillary carcinoma, follicular cancer, undifferentiation carcinoma, and medullary carcinoma, is the most common malignant tumor of the thyroid. Papillary thyroid cancer (PTC), which is less malignant and has a better prognosis, is the most common [1]. The morbidity is related to locality, race, and sexual distinction. The incidence of women is higher, and the incidence is on the rise [2]. Data in 2018 showed that the number of women suffering from thyroid cancer in China accounted for $7.7 \%$ of the total cases [3].
MicroRNAs (miRNAs) are a class of evolutionarily conserved, approximately 22-nucleotide long noncoding small RNAs. Their complementary binding with target mRNA can induce its degradation or prevent the translation of target mRNA to participate in the posttranscription regulation of target genes, which play an essential role in body development, homeostasis, and diseases [4]. miR-141$3 p$ is a member of the miR-200 cluster, and its coding gene is located on chromosome 14. At present, low abundance of miR-14-3p is found in glioblastoma, pancreatic cancer, esophageal cancer, and other tumor tissues, while highly expressing miR-141-3p is found in mammary cancer and 
other tumors [5-7]. miR-141-3p regulates the growth of cancer cells and plays different roles in different tumor cells $[8,9]$.

Propofol is one of the most commonly used narcotics during cancer resection. Relevant studies have shown that propofol may suppress the invasion of human tumor cells $[10,11]$. Zhang et al. showed that propofol can suppress the activity of cholangiocarcinoma cells and block the cell cycle to induce apoptosis [12]. At present, there are few studies on the effect of miR-141-3p in thyroid cancer. The effect of propofol on miR-141-3p expression in thyroid cancer cells has not been reported. Accordingly, our study intends to discuss the mechanism of propofol in the progression of thyroid cancer, so as to provide theoretical reference to the therapy of thyroid carcinoma.

\section{Materials and Methods}

2.1. Cell Culture and Propofol Treatment. Nthy-ori 3-1 and TPC-1 cells (from Shanghai Xuanya Biotechnology) were cultured in a $37^{\circ} \mathrm{C}$ incubator (from Thermo Fisher) containing $5 \%$ volume fraction of $\mathrm{CO}_{2}$. The medium is DMEM (from Life Technologies) involving 10\% FBS (from Life Technologies), $10 \mathrm{mg} / \mathrm{mL}$ streptomycin, and $10 \mathrm{k} \mathrm{U} / \mathrm{mL}$ penicillin (from Sangon Biotech). TPC-1 cells in the logarithmic growth period were treated with propofol (from Novartis China) and cultured overnight.

2.2. Cell Transfection. TPC-1 cells in the logarithmic growth period were seeded and incubated in 6-well plates at the density of $3 \times 10^{3}$ cells per well. When the cells' growth density reached about $50 \%$, they were transfected according to the Lipofectamine 2000 transfection reagent manual. TPC-1 cells were transfected with miR-141-3p mimic, miRNC, pcDNA-BRD4 + miR-141-3p mimic, pcDNA + miR141-3p mimic, siBRD4, and si-control (from RiboBio).

2.3. Cell Viability Assay. The cells were seeded in 96-well plates at the density of $1 \times 10^{5}$ cells per well for 72 hours, and then $50 \mu \mathrm{g}$ MTT was added to each well. After shaking and mixing, the cells were cultured in a $37^{\circ} \mathrm{C}$ incubator including $5 \%$ volume fraction of $\mathrm{CO}_{2}$ for 4 hours. After incubation, the supernatant was removed, and $100 \mu \mathrm{l}$ DMSO (from SigmaAldrich) was added into every well and oscillated for $10 \mathrm{~min}$ to completely dissolve. The absorbance of each hole was determined at $490 \mathrm{~nm}$ by using the enzyme-labeled instrument (from Molecular Devices). Cell proliferation rate $(\%)=A($ propofol $) / A($ control $) \times 100 \%$.

2.4. Cell Migration Assay. The cells were digested with trypsin (from Gibco), the cell concentration was adjusted to about $2 \times 10^{5} / \mathrm{mL}$, and then they were inoculated in a transwell well (from Corning). $100 \mu \mathrm{L}$ of TPC-1 cell suspension was added to the upper well, and $500 \mu \mathrm{L}$ of the medium (containing 10\% FBS) was added in the lower well. It was incubated for 24 hours, and the well was taken out and rinsed with PBS solution twice, then fixed with $4 \%$ paraformaldehyde for 15 minutes, and, finally, stained with crystal violet solution. After the transwell plate was dried, it was observed under an inverted microscope (from LASPEC), 5 visual fields were random choice for photographing, and the number of cells invading under the filter membrane in each visual field was counted.

2.5. Cell Invasion Assay. The cells were digested with trypsin, the cell concentration was adjusted to about $2 \times 10^{5} / \mathrm{mL}$, and then they were inoculated in a Matrigel-coated transwell well. $100 \mu \mathrm{L}$ of TPC- 1 cell suspension was added to the upper well, and $500 \mu \mathrm{L}$ of the medium (containing $10 \% \mathrm{FBS}$ ) was added in the lower well. It was incubated for 24 hours, and the well was taken out and rinsed with PBS solution twice, then fixed with $4 \%$ paraformaldehyde for 15 minutes, and, finally, stained with crystal violet solution. After the transwell plate was dried, it was observed under an inverted microscope, 5 visual fields were random choice for photographing, and the number of cells invading under the filter membrane in each visual field was counted.

2.6. Real-Time Quantitative PCR (RT-qPCR). The cells in the logarithmic growth phase were collected, total RNA was extracted from these cells with TRIzol test kits (from Thermo Fisher), and cDNA was synthesized according to the reverse transcription kit (from Invitrogen). cDNA was used as the template to perform RT-qPCR test to sense the abundance of miR-141-3p and BRD4 in these cells. The relative abundance was calculated by the $2^{-\Delta \Delta \mathrm{Ct}}$ method. The internal reference is U6. The sequence of primers used in this experiment (from Sangon Biotech) is shown in Table 1.

2.7. Dual-Luciferase Reporter Gene Method. The bioinformatics online database TargetScan was used to forecast the targeted gene of miR-141-3p. The results showed that there was a targeted binding site between the $3^{\prime}$-UTR of BRD4 and miR-141-3p, indicating that BRD4 is probably the targeting gene of miR-141-3p. The luciferase recombinant vectors containing wild-type BRD4 3'-UTR (BRD4-Wt) and mutant BRD4 3'-UTR (BRD4-Mut) were amplified and constructed, respectively. The BRD4-Wt or BRD4-Mut recombinant vectors were cotransfected with miR-141-3p mimic and miR-con into TPC-1 cells, respectively. After 48 hours, the luciferase activity was measured using the dual-luciferase activity detection kit (from Promega) to calculate the relative fluorescence activity of cells.

2.8. Western Blotting. Each group of cells was taken and added cell lysate (from Beyotime Biotechnology) and then incubated on ice for 30 minutes. The protein in the cells was collected, and the total protein was quantified with the BCA protein detection kit (from Beyotime Biotechnology). $50 \mu \mathrm{g}$ protein sample was taken, and the protein was separated with $12 \%$ sodium lauryl sulfate-polyacrylamide gel, transferred to the nitrocellulose membrane (from Sigma), and blocked for 1 hour. Protein primary antibody (from Abcam) 
TAble 1: Primer sequences of RT-qPCR.

\begin{tabular}{lc}
\hline Gene & \multicolumn{1}{c}{ Sequence } \\
\hline \multirow{2}{*}{ miR-141-3p } & F: $5^{\prime}$-GCGGCGGTAACACTGTCTGG-3' \\
& R: $5^{\prime}$-AACGCTTCACGAATTTGCGT-3 ${ }^{\prime}$ \\
\hline \multirow{2}{*}{ BRD4 } & F: $5^{\prime}$-GCACAATCAAGTCTAAACTGGAG-3' \\
& R: $5^{\prime}$-TCATGGTCAGGAGGGTTGTAC-3 ${ }^{\prime}$ \\
\hline \multirow{2}{*}{ U6 } & F: $5^{\prime}$-GCTTCGGCAGCACATATACTAAAAT-3' \\
& R: $5^{\prime}$-CGCTTCACGAATTTGCGTGTCAT-3' \\
\hline
\end{tabular}

was added and incubated overnight at $4^{\circ} \mathrm{C}$. Secondary antibodies (from Abcam) were added at room temperature on the next day, incubated for 1 hour, and placed in the gel imaging system for exposure, and Quantity One software was used to analyze the gray value of protein bands.

2.9. Statistical Analysis. Statistical software SPSS 17.0 and GraphPad Prism 8.0.2 were used for statistical analysis of experimental data. Statistical data were expressed as mean \pm standard deviation $(x \pm s)$, $t$-test. The count data were described by the utilization rate (\%). $P<0.05$ represents that the difference had a statistical significance.

\section{Results}

3.1. Propofol Suppresses Proliferation, Migration, and Invasion of Thyroid Cancer Cells. In this experiment, MTT method and transwell method were used to test the influences of propofol on the phenotype of thyroid cancer cells. From results compared with the control group $(0 \mu \mathrm{g} / \mathrm{L})$, the activity of TPC-1 cells in the propofol group was significantly decreased (Figure 1), and their migration and invasion were also significantly inhibited (Figure 2).

3.2. miR-141-3p Suppresses Proliferation, Migration, and Invasion of Thyroid Cancer Cells. The abundance of miR-141$3 \mathrm{p}$ in thyroid cancer cells was detected by RT-qPCR. We found that the abundance of miR-141-3p in TPC-1 cells was significantly decreased than that in Nthy-ori 3-1 cells $(P<0.001$, Figure 3(a)). In order to study the influence of miR-141-3p on the activity of TPC-1 cells, we used miR-141$3 \mathrm{p}$ mimic to induce the expression of miR-141-3p in TPC-1 cells. As shown in Figure 3(b) $(P<0.001)$, the abundance of miR-141-3p in TPC-1 cells transfected with miR-141-3p mimic was obviously superior than that in the negative control, indicating that the cells' excessive expression miR141-3p had been successfully established. MTT and transwell results showed that overexpression of miR-141-3p obviously inhibited the phenotype of TPC-1 cells (all $P<0.05$, Figures 3(c)-3(e)).

3.3. miR-141-3p Expression Is Promoted by Propofol. The expression of miR-141-3p in TPC-1 cells treated with propofol for 24 hours was detected by RT-qPCR, and we found that the expression of miR-141-3p in TPC-1 cells was obviously increased $(P<0.001$, Figure 4$)$.

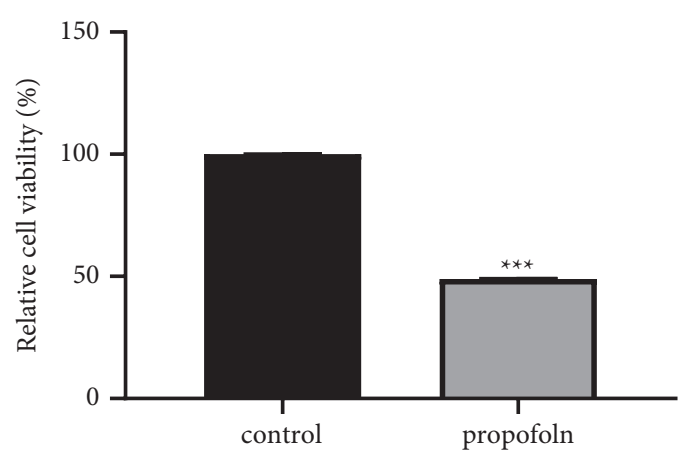

Figure 1: Effect of propofol on proliferation of TPC-1 cells $\left({ }^{* * *} P<0.001\right)$.

3.4. BRD4 Is the Targeting Gene of miR-141-3p. TargetScan prediction result displayed that there was a specific binding site between miR-141-3p and BRD4 $3^{\prime}$-UTR (Figure 5(a)). After wild-type BRD4 luciferase expression vector WT-BRD4 was cotransfected with miR-141-3p mimics or miR-con into TPC-1 cells, respectively, the luciferase activities of TPC-1 cells in the miR-141-3p group were obviously decreased than those in miR-con $(P<0.001)$. However, after cotransfection of mutant BRD4 luciferase expression vector MUT-BRD4 with miR-141-3p mimic or miR-con on TPC-1 cells, the difference was not statistically significant in luciferase activity between the miR-141-3p group and miR-con $(P>0.05)$ (Figure $5(b))$.

3.5. Propofol Suppresses the SHH and PI3K/AKT Pathways. According to the analysis of western blotting results, the protein abundance of SHH, GLI1, p-PI3K, and p-AKT was inhibited after propofol treatment for 24 hours $(P<0.01$, Figure 6(a)).

3.6. SHH and PI3K/AKT Pathways Are Regulated by miR-141$3 p$ Expression. As shown in Figure 6(b), the protein abundance of SHH, GLI1, p-PI3K, and p-AKT in TPC-1 cells after overexpression of miR-141-3p was lower than that in the untransfected and transfected miR-NC groups $(P<0.01)$.

3.7. SHH and PI3K/AKT Pathways Are Regulated by BRD4 Expression. As shown in Figure 6(c), compared with the cells in the untransfected and transfected si-control groups, the protein abundance of $\mathrm{SHH}, \mathrm{GLI} 1, \mathrm{p}-\mathrm{PI} 3 \mathrm{~K}$, and $\mathrm{p}-\mathrm{AKT}$ in TPC-1 cells decreased after BRD4 was silenced $(P<0.001)$.

3.8. BRD4 Overexpression Partially Reversed the Influences of miR-141-3p on TPC-1 Cell Activity. As shown in Figure 7, compared with the miR-141-3p+pcDNA group, the phenotype of TPC-1 cells in the miR-141-3p + pcDNA-BRD4 group was significantly promoted $(P<0.05)$.

\section{Discussion}

Propofol is one of the frequently used narcotics for cancer resection. Since Mammoto et al. first proposed in 2002 that 

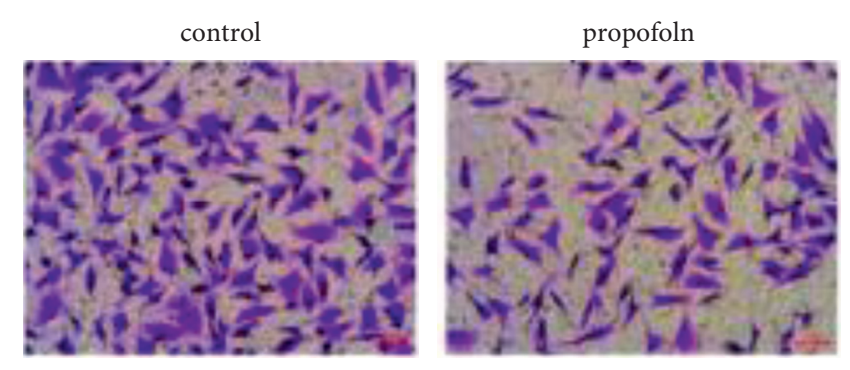

(a)
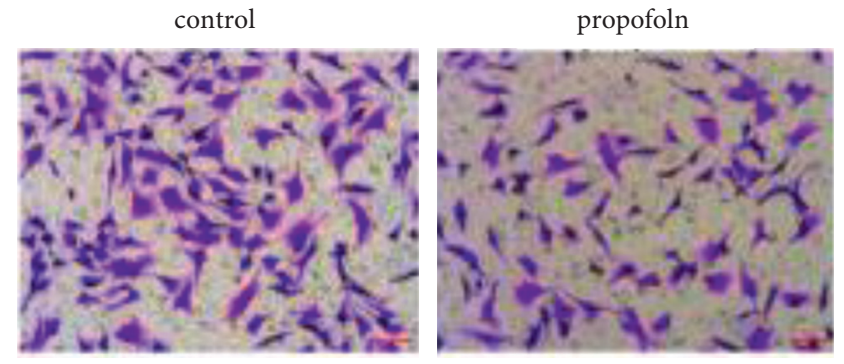

(b)
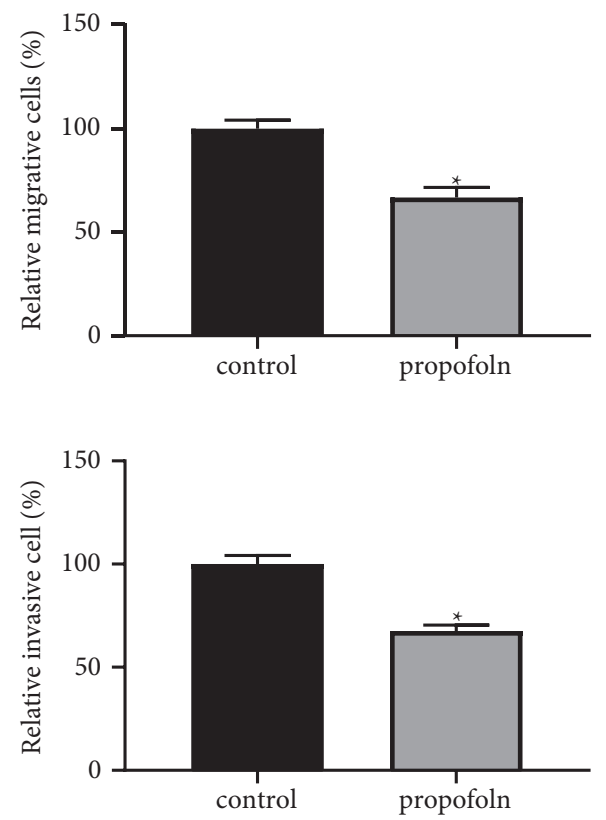

FIGURE 2: Effect of propofol on migration and invasion of TPC-1 cells. Propofol can inhibit the migration (a) and invasion (b) of TPC-1 cells $\left({ }^{*} P<0.05\right)$.

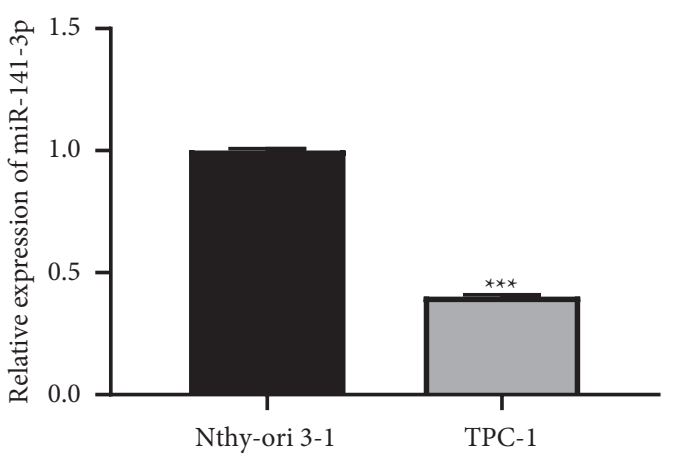

(a)

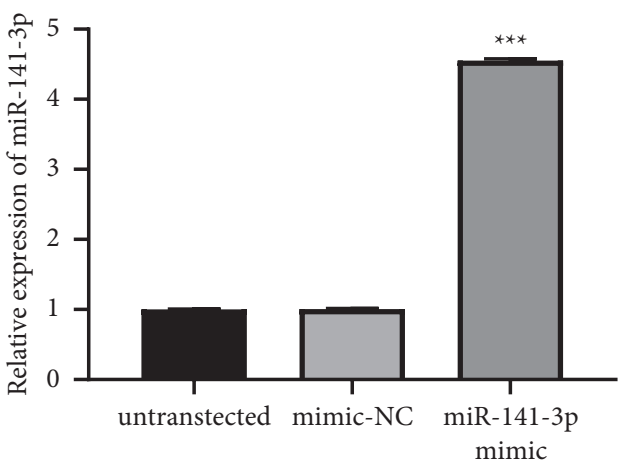

(b)

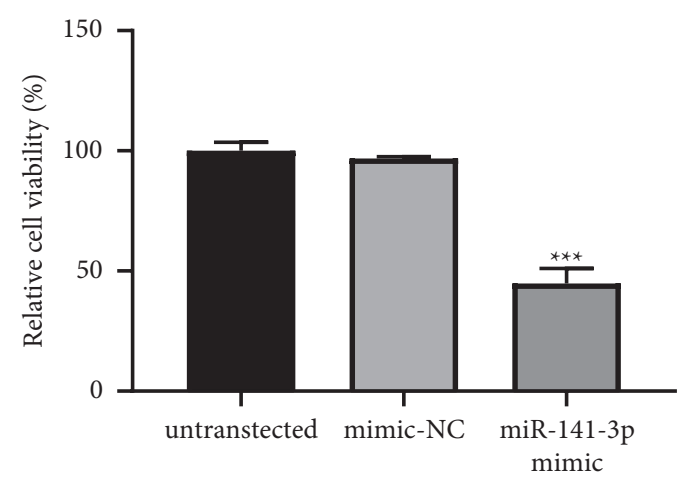

(c)

Figure 3: Continued. 
untransfected

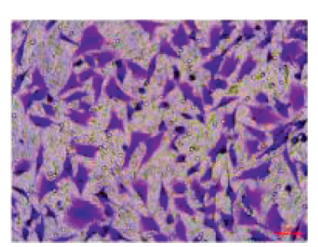

mimic-NC

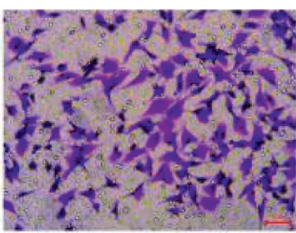

miR-141-3p mimic

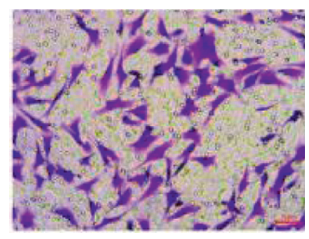

(d)

miR-141-3p mimic

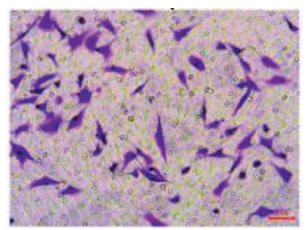

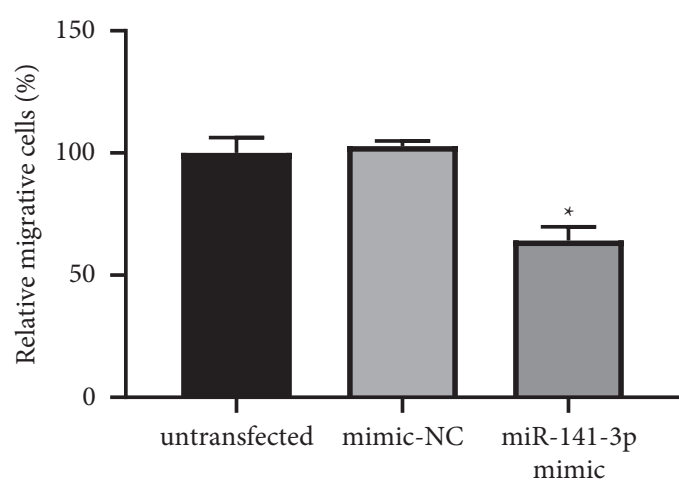

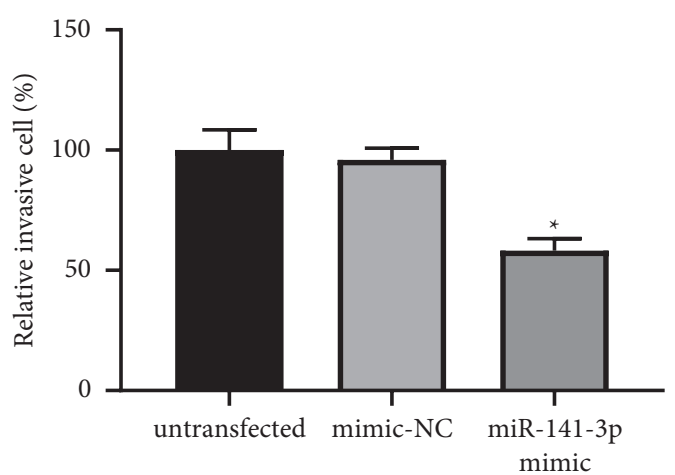

(e)

FIgURE 3: The abundance of miR-141-3p in TPC-1 cells and its influence on the biological behavior of cancer cells. (a) Compared with Nthyori 3-1 cells, the expression of miR-141-3p in TPC-1 cells was significantly decreased $\left({ }^{* * *} P<0.001\right)$. (b) The abundance of miR-141-3p was successfully increased in TPC-1 cells by using miR-141-3p mimics $\left({ }^{* * *} P<0.001\right)$. (c-e) Overexpression of miR-141-3p significantly inhibited the phenotype of TPC- 1 cells $\left({ }^{*} P<0.05\right.$ and $\left.{ }^{* * *} P<0.001\right)$.

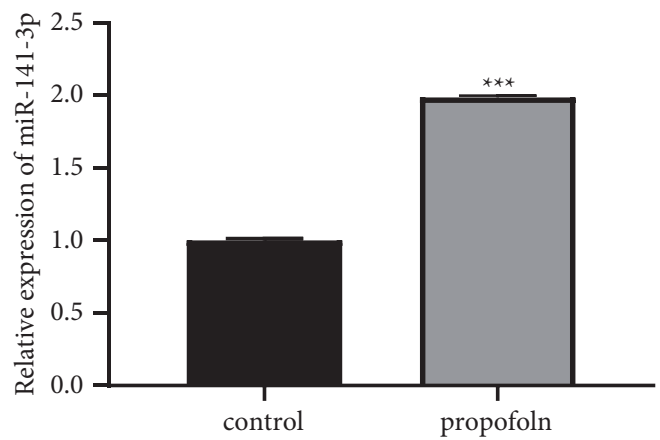

Figure 4: Propofol promotes miR-141-3p expression in TPC-1 cells $\left({ }^{* *} P<0.001\right)$.

clinical related concentrations of propofol could inhibit the invasion of human cancer cells (cervical cancer HeLa cells, fibrosarcoma HT1080 cells, osteosarcoma HOS cells, and melanoma RPMI-7951 cells) [13], its effect on tumor cells and its mechanism have become a rapidly developing topic and gradually attracted extensive attention. Related studies have shown that propofol can inhibit the invasion and metastasis of esophageal squamous cell carcinoma cells by downregulating the expression of SOX4 [14]. In addition, propofol can also inhibit invasion and angiogenesis and induce apoptosis of esophageal cancer EC-1 cells in vitro by regulating the expression of S100A4 [15]. Chen et al. showed that propofol could inhibit the migration of pancreatic cancer cells by inhibiting NMDA receptors [16]. In addition, propofol can also inhibit the proliferation, invasion, and metastasis of pancreatic cancer cells and induce apoptosis of tumor cells by upregulating the expression of miR-133a and miR-21 [17, 18]. Li et al. found that propofol can inhibit the phenotype of PTC cells by inhibiting the activation of the $\mathrm{NF}-\kappa \mathrm{B}$ pathway and $\mathrm{Wnt} / \beta$-catenin [19]. This is consistent 


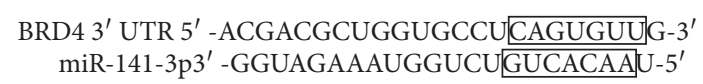

(a)

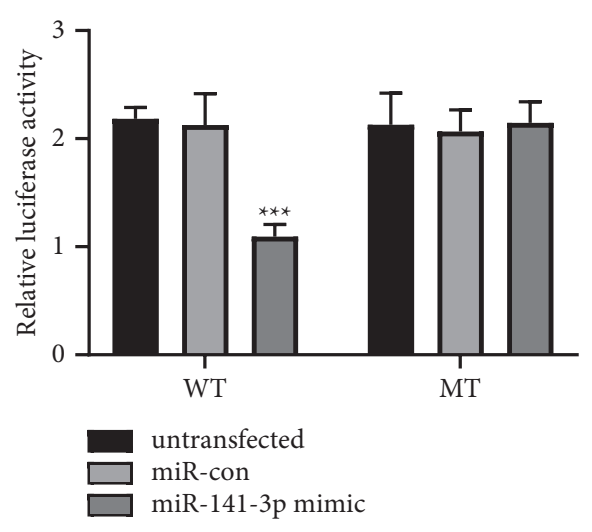

(b)

FIGURE 5: BRD4 is a targeting gene of miR-141-3p in TPC-1 cells. (a) A complement sequence of miR-141-3p is found in the $3^{\prime}$-UTR of BRD4. (b) Dual-luciferase reporter gene analysis proved the interaction of miR-141-3p and BRD4 ( $\left.{ }^{* * *} P<0.001\right)$.
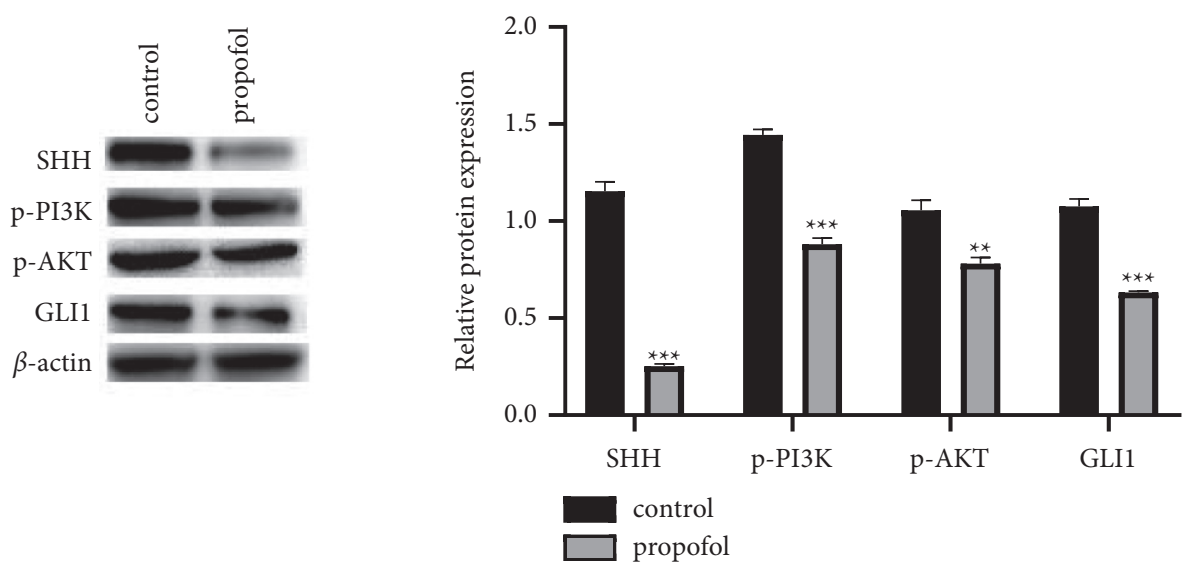

(a)
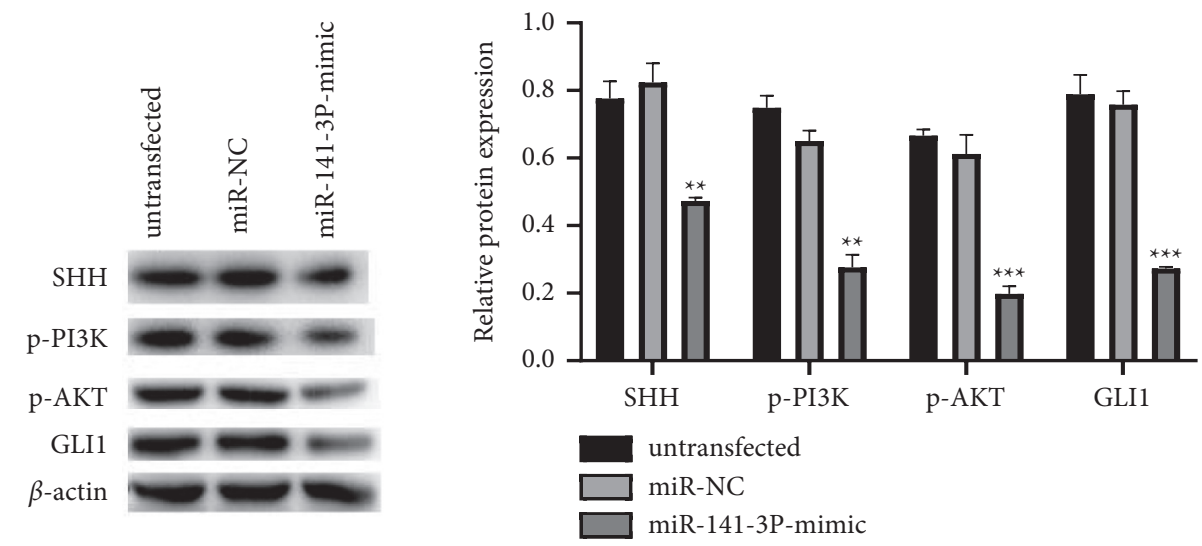

(b)

FIGURE 6: Continued. 

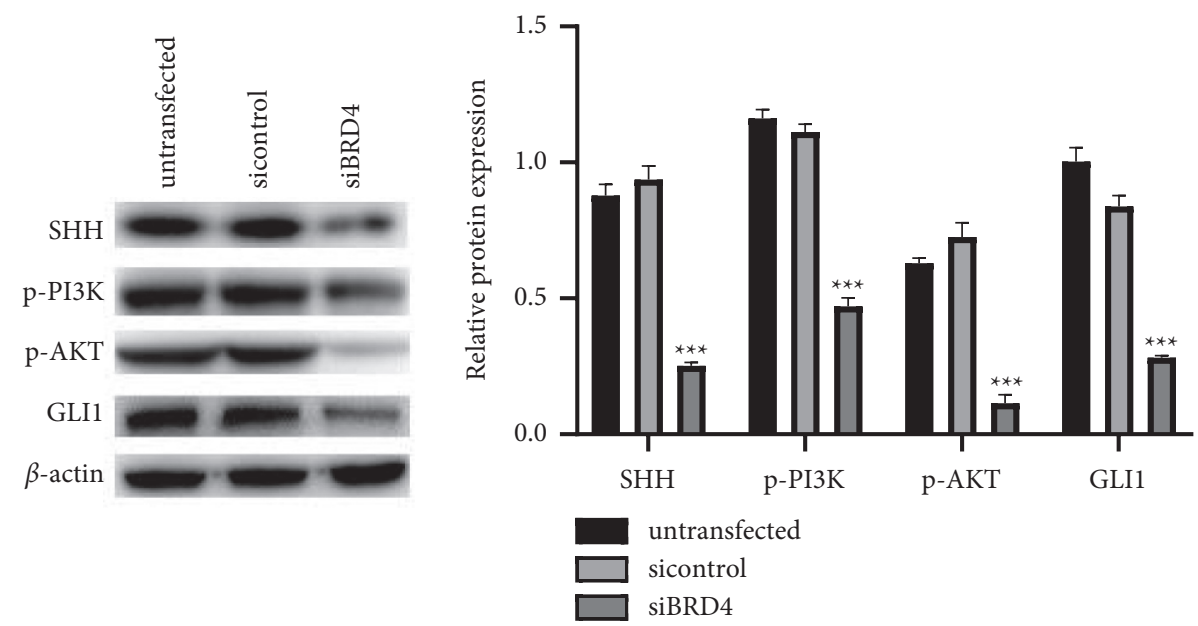

(c)

FIGURE 6: Influences of propofol (a), miR-141-3p (b), and BRD4 (c) on the expressions of key proteins related to SHH and PI3K/AKT pathways $\left({ }^{* *} P<0.01\right.$ and $\left.{ }^{* * *} P<0.001\right)$.

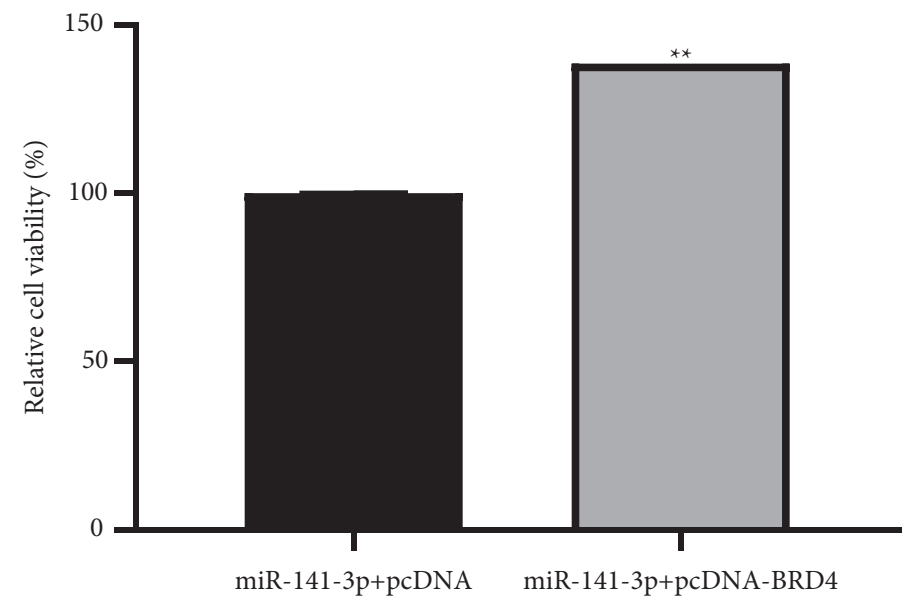

(a)

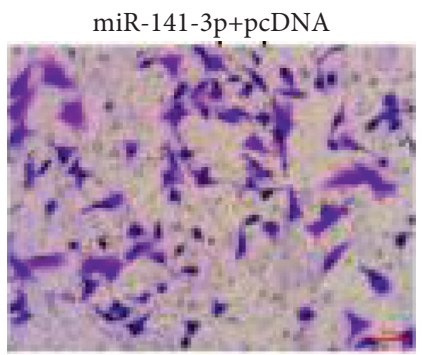

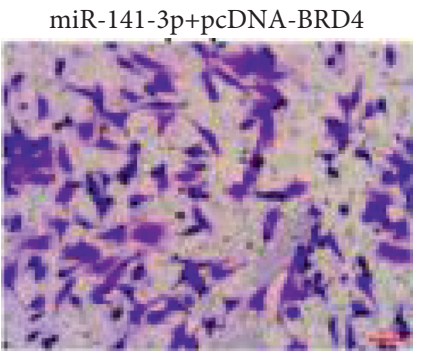

(b)

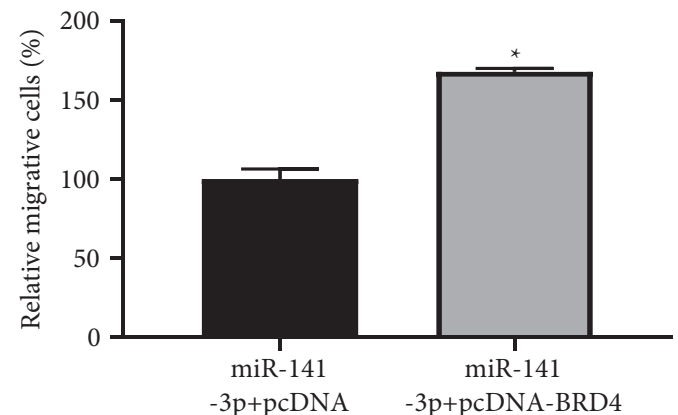

$-3 p+p c D N A$

FIgURe 7: Continued. 


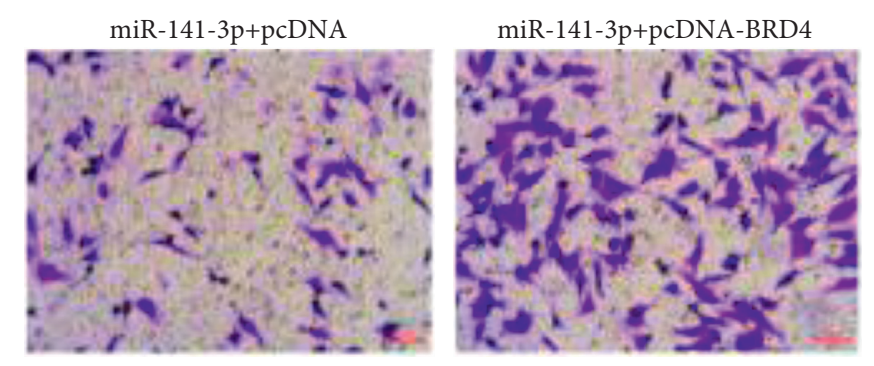

(c)

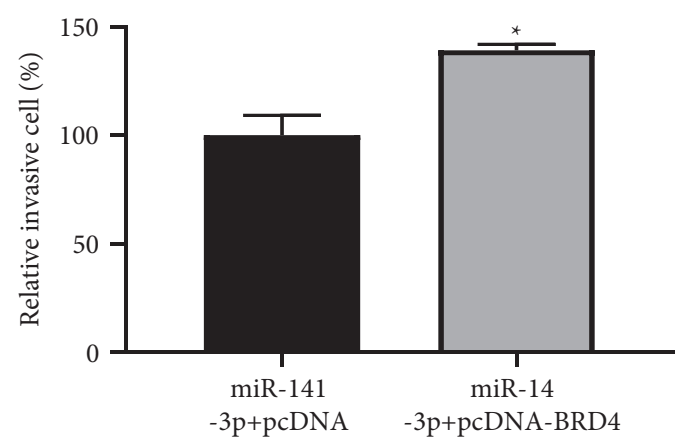

FIgURE 7: Cell proliferation (a), migration (b), and invasion (c) are all promoted by overexpression of BRD4 in TPC-1 cells $\left({ }^{*} \mathrm{P}<0.05\right)$.

with the results of this experiment; we found that the phenotype of TPC-1 cells treated with propofol was significantly inhibited, suggesting that propofol had a certain restraining effect on the malignant behavior of thyroid tumor cells.

Relevant research studies have already shown that the antitumor influence of propofol perhaps is closely in connection with miRNA it regulates. For example, propofol can inhibit the proliferation of mammary cancer MCF-7 cells by downregulating the expression of miR-21 [20] and can also inhibit the activity of melanoma cells by adjusting miR-137 and FGF9 [21]. miRNAs play important roles in development, cell differentiation, hematopoietic function, cell apoptosis, growth, and immune system. Many human diseases including cancer, autoimmune diseases, and chronic diseases are related to the abnormal regulation of miRNA. Dong et al. found that miR141 was downregulated in thyroid tumor tissue [22], which equates with our study. We found that propofol could increase the abundance of miR-141-3p in TPC-1 cells. To verify the role of miR-141-3p in thyroid cancer, we overexpressed miR-141$3 p$ in thyroid cancer cells. The results showed that overexpression of miR-141-3p inhibited the phenotype of TPC-1 cells. It is speculated that miR-141-3p may play an important role in the antithyroid cancer mechanism of propofol.

BRD4 is one of the members of the bromodomain and extraterminal domain (BET) family, which is an essential epigenetic regulator of gene transcription and cancer development. As one of them, BRD4 can regulate various characteristics of cancer cells by adjustment of the expression and activity of cancer promoters, including drug resistance, apoptosis, cell transformation, proliferation, and invasion [23]. BRD4 is overexpressed in a variety of parenchymatous tumors, including pancreatic cancer, mammary cancer, and colorectal cancer, and its expression inhibition can hinder the invasion and proliferation of these tumor cells $[24,25]$. In this study, we predicted and proved that BRD4 is a targeted gene of miR-1413 p by bioinformatics, and overexpression of BRD4 can partially reverse the inhibitory effect of miR-141-3p on the phenotype of thyroid cancer cells. It is speculated that miR-141-3p may inhibit the malignant behavior of thyroid cancer cells by regulating the abundance of BRD4.

$\mathrm{SHH}$ and PI3K/AKT are both pathways that play important roles in cancer progression. $\mathrm{SHH}$ is involved in tumor metastasis in basal cell carcinoma, ovarian cancer, cervical cancer, breast cancer, gastric cancer, and pancreatic cancer and is related to drug resistance and survival of cancer [26-31]. Among the disordered signaling pathways, $\mathrm{PI} 3 \mathrm{~K} / \mathrm{AKT}$ pathway is the most frequently changed signaling pathway. AKT is a Ser/Thr protein kinase and an important node in the PI3K signaling pathway. It has three different subtypes, AKT1, AKT2, and AKT3, which are closely related to the development of human cancer [32, 33]. Previous research studies have shown that BRD4 can promote the phenotype of thyroid cancer cells through the SHH pathway. In addition, downregulation of BRD4 in GBC cells can induce apoptosis through the PI3K/AKT pathway. In the current study, we obtained the conclusion that propofol treatment, overexpression of miR-141-3p, and silencing $\mathrm{BRD} 4$ can downregulate the abundance of $\mathrm{SHH}$ and $\mathrm{PI} 3 \mathrm{~K} /$ AKT pathway-related proteins SHH, GLI1, p-PI3K, and p-AKT and inhibit SHH and PI3K/AKT signal pathways' activation.

In summary, our study confirmed the inhibition of propofol for the malignant behavior of thyroid tumor. Its antitumor effect may be achieved by regulating miR-141-3p to target BRD4 affecting the activity of SHH and PI3K/AKT signaling pathways. However, there are many related factors that regulate the behavior of thyroid cancer cells; this study still has certain limitations. The specific mechanism of propofol's inhibitory effect on thyroid cancer cells needs to be further studied.

\section{Data Availability}

The data used to support the findings of this study are available from the corresponding author upon reasonable request.

\section{Conflicts of Interest}

The authors declare no conflicts of interest.

\section{Authors' Contributions}

Heming Zhang and Mingtao Tan contributed equally to this article. 


\section{References}

[1] L. G. T. Morris, R. M. Tuttle, and L. Davies, "Changing trends in the incidence of thyroid cancer in the United States," JAMA Otolaryngology-Head \& Neck Surgery, vol. 142, no. 7, pp. 709-711, 2016 Jul 1.

[2] W. Chen, R. Zheng, P. D. Baade et al., "Cancer statistics in China, 2015," CA: A Cancer Journal for Clinicians, vol. 66, no. 2, pp. 115-132, 2016 Mar-Apr.

[3] R.-M. Feng, Y.-N. Zong, S.-M. Cao, and R.-H. Xu, "Current cancer situation in China: good or bad news from the 2018 Global Cancer Statistics?" Cancer Communications, vol. 39, no. 1, p. 22, 2019 Apr 29.

[4] P. Paul, A. Chakraborty, D. Sarkar et al., "Interplay between miRNAs and human diseases," Journal of Cellular Physiology, vol. 233, no. 3, pp. 2007-2018, 2018 Mar.

[5] M. Wang, M. Hu, Z. Li, D. Qian, B. Wang, and D. X. Liu, "miR-141-3p functions as a tumor suppressor modulating activating transcription factor 5 in glioma," Biochemical and Biophysical Research Communications, vol. 490, no. 4, pp. 1260-1267, 2017 Sep 2.

[6] J. Sun and Y. Zhang, "LncRNA XIST enhanced TGF- $\beta 2$ expression by targeting miR-141-3p to promote pancreatic cancer cells invasion," Bioscience Reports, vol. 39, no. 7, Article ID BSR20190332, 2019 Jul 2.

[7] P. Li, T. Xu, X. Zhou et al., "Downregulation of miRNA-141 in breast cancer cells is associated with cell migration and invasion: involvement of ANP32E targeting," Cancer Medicine, vol. 6, no. 3, pp. 662-672, 2017 Mar.

[8] C. Liu, R. Liu, D. Zhang et al., "MicroRNA-141 suppresses prostate cancer stem cells and metastasis by targeting a cohort of pro-metastasis genes," Nature Communications, vol. 8, no. 1, p. 14270, 2017 Jan 23.

[9] G. Lou, X. Dong, C. Xia et al., "Direct targeting sperm-associated antigen 9 by miR-141 influences hepatocellular carcinoma cell growth and metastasis via JNK pathway," Journal of Experimental \& Clinical Cancer Research, vol. 35, no. 1, p. 14, 2016 Jan 21.

[10] Z. Peng and Y. Zhang, "Propofol inhibits proliferation and accelerates apoptosis of human gastric cancer cells by regulation of microRNA-451 and MMP-2 expression," Genetics and Molecular Research, vol. 15, no. 2, 2016 Apr 4.

[11] C. Yang, J. Gao, N. Yan et al., "Propofol inhibits the growth and survival of gastric cancer cells in vitro through the upregulation of ING3," Oncology Reports, vol. 37, no. 1, pp. 587-593, 2017 Jan.

[12] Z. Zhang, M. Zang, S. Wang, and C. Wang, "Effects of propofol on human cholangiocarcinoma and the associated mechanisms ," Experimental and Therapeutic Medicine, vol. 17, no. 1, pp. 472-478, 2019 Jan.

[13] T. Mammoto, M. Mukai, A. Mammoto et al., "Intravenous anesthetic, propofol inhibits invasion of cancer cells," Cancer Letters, vol. 184, no. 2, pp. 165-170, 2002 Oct 28.

[14] C.-L. Zhou, J.-J. Li, and P. Ji, "Propofol suppresses esophageal squamous cell carcinoma cell migration and invasion by down-regulation of sex-determining region Y-box 4 (SOX4)," Medical Science Monitor, vol. 23, pp. 419-427, 2017 Jan 24.

[15] X. G. Guo, S. Wang, Y. B. Xu, and J. Zhuang, "Propofol suppresses invasion, angiogenesis and survival of EC-1 cells in vitro by regulation of S100A4 expression," European Review for Medical and Pharmacological Sciences, vol. 19, no. 24, pp. 4858-4865, 2015 Dec.

[16] X. Chen, Q. Wu, L. You, S. Chen, M. Zhu, and C. Miao, "Propofol attenuates pancreatic cancer malignant potential via inhibition of NMDA receptor," European Journal of Pharmacology, vol. 795, pp. 150-159, 2017 Jan 15.

[17] Z. T. Wang, H. Y. Gong, F. Zheng, D. J. Liu, and T. L. Dong, "Propofol suppresses proliferation and invasion of pancreatic cancer cells by upregulating microRNA-133a expression," Genetics and Molecular Research, vol. 14, no. 3, pp. 7529-7537, 2015 Jul 6.

[18] Z. Liu, J. Zhang, G. Hong, J. Quan, L. Zhang, and M. Yu, "Propofol inhibits growth and invasion of pancreatic cancer cells through regulation of the miR-21/Slug signaling pathway," American Journal of Tourism Research, vol. 8, no. 10, pp. 4120-4133, 2016 Oct 15.

[19] M. Li, L. Qu, F. Chen, and X. Zhu, "Propofol upregulates miR$320 \mathrm{a}$ and reduces HMGB1 by downregulating ANRIL to inhibit PTC cell malignant behaviors," Pathology, Research \& Practice, vol. 216, no. 4, p. 152856, 2020 Apr.

[20] Q. Du, X. Zhang, X. Zhang, M. Wei, H. Xu, and S. Wang, "Propofol inhibits proliferation and epithelial-mesenchymal transition of MCF-7 cells by suppressing miR-21 expression," Artificial Cells, Nanomedicine, and Biotechnology, vol. 47, no. 1, pp. 1265-1271, 2019 Dec.

[21] H. Yu, M. Ma, X. Wang, Z. Zhou, R. Li, and Q. Guo, "Propofol suppresses proliferation, invasion, and migration of human melanoma cells via regulating microRNA-137 and fibroblast growth factor 9," Journal of Cellular Physiology, vol. 234, no. 12, pp. 23279-23288, 2019 Dec.

[22] S. Dong, X. Meng, S. Xue, Z. Yan, P. Ren, and J. Liu, "microRNA-141 inhibits thyroid cancer cell growth and metastasis by targeting insulin receptor substrate 2," American Journal of Tourism Research, vol. 8, no. 3, pp. 1471-1481, 2016 Mar 15.

[23] X. Jin, Y. Yan, D. Wang et al., "DUB3 promotes BET inhibitor resistance and cancer progression by deubiquitinating BRD4," Molecular Cell, vol. 71, no. 4, pp. 592-605, 2018 Aug 16.

[24] V. K. Mishra, F. Wegwitz, R. L. Kosinsky et al., "Histone deacetylase class-I inhibition promotes epithelial gene expression in pancreatic cancer cells in a BRD4- and MYCdependent manner," Nucleic Acids Research, vol. 45, no. 11, pp. 6334-6349, 2017 Jun 20.

[25] Y. Wang, Y. Sui, Q. Zhu, and X. Sui, "Hsa-miR-599 suppresses the migration and invasion by targeting BRD4 in breast cancer," Oncology Letters, vol. 14, no. 3, pp. 3455-3462, 2017 Sep.

[26] A. M. Skoda, D. Simovic, V. Karin, V. Kardum, S. Vranic, and L. Serman, "The role of the Hedgehog signaling pathway in cancer: a comprehensive review," Bosnian Journal of Basic Medical Sciences, vol. 18, no. 1, pp. 8-20, 2018 Feb 20.

[27] H. Li, J. Li, and L. Feng, "Hedgehog signaling pathway as a therapeutic target for ovarian cancer," Cancer Epidemiology, vol. 40, pp. 152-157, 2016 Feb.

[28] C. Huang, H. Lu, J. Li et al., "SOX2 regulates radioresistance in cervical cancer via the hedgehog signaling pathway," Gynecologic Oncology, vol. 151, no. 3, pp. 533-541, 2018 Dec.

[29] N. Riobo-Del Galdo, Á. Lara Montero, and E. Wertheimer, "Role of hedgehog signaling in breast cancer: pathogenesis and therapeutics," Cells, vol. 8, no. 4, p. 375, 2019 Apr 25.

[30] D. H. Lee, S. Y. Lee, and S. C. Oh, "Hedgehog signaling pathway as a potential target in the treatment of advanced gastric cancer," Tumour biology: The Journal of the International Society for Oncodevelopmental Biology and Medicine, vol. 39, no. 6, Article ID 1010428317692266, 2017 Jun.

[31] E. Klieser, S. Swierczynski, C. Mayr et al., "Differential role of Hedgehog signaling in human pancreatic (patho-) physiology: 
an up to date review," World Journal of Gastrointestinal Pathophysiology, vol. 7, no. 2, pp. 199-210, 2016 May 15.

[32] S. Bugide, V. K. Gonugunta, V. Penugurti, V. L. Malisetty, R. K. Vadlamudi, and B. Manavathi, "HPIP promotes epithelial-mesenchymal transition and cisplatin resistance in ovarian cancer cells through PI3K/AKT pathway activation," Cellular Oncology, vol. 40, no. 2, pp. 133-144, 2017 Apr.

[33] M. K. Ediriweera, K. H. Tennekoon, and S. R. Samarakoon, "Role of the PI3K/AKT/mTOR signaling pathway in ovarian cancer: biological and therapeutic significance," Seminars in Cancer Biology, vol. 59, pp. 147-160, 2019 Dec. 